\title{
A NEW APPROACH TO CATALAN NUMBERS USING DIFFERENTIAL EQUATIONS
}

\author{
TAEKYUN KIM AND DAE SAN KIM
}

\begin{abstract}
In this paper, we introduce two differential equations arising from the generating function of the Catalan numbers which are 'inverses' to each other in some sense. From these differential equations, we obtain some new and explicit identities for Catalan and higher-order Catalan numbers. In addition, by other means than differential equations we also derive some interesting identities involving Catalan numbers which are of arithmetic and combinatorial nature.
\end{abstract}

\section{INTRODUCTION}

The Catalan numbers $C_{n}$ were first introduced by the Mongolian mathematician Ming Antu in around 1730, even though they were named after the Belgian mathematician Eugène Charles Catalan (1814-1894). Indeed, Ming Antu obtained a number of trigonometric expressions involving Catalan numbers such as

$$
\sin 2 \theta=2 \sin \theta-\sum_{n=1}^{\infty} \frac{C_{n-1}}{4^{n-1}} \sin ^{2 n+1} \theta=2 \sin \theta-\sin ^{3} \theta-\frac{1}{4} \sin ^{5} \theta-\frac{1}{8} \sin ^{7} \theta-\cdots
$$

(see [2 4, 6, 10 14]). The Catalan numbers can be given explicitly in terms of binomial coefficients. Namely, for $n \geq 0$,

$$
C_{n}=\frac{1}{n+1}\left(\begin{array}{c}
2 n \\
n
\end{array}\right)=\prod_{k=2}^{n} \frac{n+k}{k} .
$$

They satisfy the recurrence relations

$$
C_{0}=1, C_{n}=\sum_{m=0}^{n-1} C_{m} C_{n-1-m}, \quad(n \geq 1) .
$$

The Catalan numbers are also given by the generating function

$$
\frac{2}{1+\sqrt{1-4 t}}=\sum_{n=0}^{\infty} C_{n} t^{n}=\sum_{n=0}^{\infty} \frac{1}{n+1}\left(\begin{array}{c}
2 n \\
n
\end{array}\right) t^{n} .
$$

The Catalan numbers form the sequence of positive integers

$1,1,2,5,14,42,132,429,1430,4862,16796,58786,208012, \cdots$

which is asymptotic to $\frac{4^{n}}{n^{\frac{3}{2}} \sqrt{\pi}}$, as $n$ tends to $\infty$, and appears in various counting problems. For example, $C_{n}$ is the number of Dyck words of length $2 n$, the number of balanced $n$ pairs of parentheses, the number of mountain ranges you can form

2010 Mathematics Subject Classification : 05A19, 11B37, 11B83, 34A34.

Key words and phrases: Catalan numbers, differential equations. 
with $n$ upstrokes and downstrokes that all stay above the original line, the number of diagonal-avoiding paths of length $2 n$ from the upper left corner to the lower right corner in a grid of $n \times n$ squares, and the number of ways $n+1$ factors can be completely parenthesized (see [1-4, 10, 11]).

It is also the number of ways an $(n+2)$-gon can be cut into $n$ triangles, the number of permutations of $\{1,2, \cdots, n\}$ that avoid the pattern 123 , the number of ways to tile a stairstep shape of height $n$ with $n$ rectangles, etc (see [10 13]).

In [8], T. Kim initiated a fascinating idea of using ordinary differential equations as a method of obtaining new identities for special polynomials and numbers. Namely, a family of nonlinear differential equations were derived, which are indexed by positive integers and satisfied by the generating function of the Frobenius-Euler numbers. Then, they were used in order to obtain an interesting identity, expressing higher-order Frobenius-Euler numbers in terms of (ordinary) Frobenius-Euler numbers (see [5, 7, 9, 15]).

This method turned out to be very fruitful and can be applied to many interesting special polynomials and numbers (see [7-9]). For example, linear differential equations are derived for Bessel polynomials, Changhee polynomials, actuarial polynomials, Meixner polynomials of the first kind, Poisson-Charlier polynomials, Laguerre polynomials, Hermite polynomials, and Stirling polynomials, while nonlinear ones are obtained for Bernoulli numbers of the second, Boole numbers, Chebyshev polynomials of the first, second, third, and fourth kind, degenerate Euler numbers, degenerate Eulerian polynomials, Korobov numbers, and Legendre polynomials (see $[1,[5,7,9,[15])$.

In this paper, we introduce two differential equations arising from the generating function of the Catalan numbers which are 'inverses' to each other in some sense. From these differential equations, we obtain some new and explicit identities for Catalan and higher-order Catalan numbers. In addition, by other means than differential equations we also derive some interesting identities involving Catalan numbers which are of arithmetic and combinatorial nature.

\section{Differential equations associated with Catalan numbers}

Let

$$
C=C(t)=\frac{2}{1+\sqrt{1-4 t}}
$$

Then, by (2.1), we have

$$
\begin{aligned}
C^{(1)} & =\frac{d}{d t} C(t)=(1-4 t)^{-\frac{1}{2}} 4(1+\sqrt{1-4 t})^{-2} \\
& =(1-4 t)^{-\frac{1}{2}} C^{2}
\end{aligned}
$$

and

$$
\begin{aligned}
C^{(2)} & =\frac{d}{d t} C^{(1)}=2(1-4 t)^{-\frac{3}{2}} C^{2}+2(1-4 t)^{-\frac{1}{2}} C C^{(1)} \\
& =2(1-4 t)^{-\frac{3}{2}} C^{2}+2(1-4 t)^{-\frac{1}{2}} C\left\{(1-4 t)^{-\frac{1}{2}} C^{2}\right\} \\
& =2(1-4 t)^{-\frac{3}{2}} C^{2}+2(1-4 t)^{-\frac{2}{2}} C^{3}
\end{aligned}
$$


So, we are led to put

$$
C^{(N)}=\sum_{i=1}^{N} a_{i}(N)(1-4 t)^{-\frac{2 N-i}{2}} C^{i+1},
$$

where $N=1,2,3, \cdots$. From (2.4), we obtain

$$
\begin{aligned}
C^{(N+1)}= & \frac{d}{d t} C^{(N)} \\
= & \sum_{i=1}^{N} 2(2 N-i) a_{i}(N)(1-4 t)^{-\frac{2 N+2-i}{2}} C^{i+1} \\
& +\sum_{i=1}^{N}(i+1) a_{i}(N)(1-4 t)^{-\frac{2 N-i}{2}} C^{i} C^{(1)} \\
= & \sum_{i=1}^{N} 2(2 N-i) a_{i}(N)(1-4 t)^{-\frac{2 N+2-i}{2}} C^{i+1} \\
& +\sum_{i=1}^{N}(i+1) a_{i}(N)(1-4 t)^{-\frac{2 N-i}{2}} C^{i}\left\{(1-4 t)^{-\frac{1}{2}} C^{2}\right\} \\
= & \sum_{i=1}^{N} 2(2 N-i) a_{i}(N)(1-4 t)^{-\frac{2 N+2-i}{2}} C^{i+1} \\
& +\sum_{i=1}^{N}(i+1) a_{i}(N)(1-4 t)^{-\frac{2 N+1-i}{2}} C^{i+2} \\
= & \sum_{i=1}^{N} 2(2 N-i) a_{i}(N)(1-4 t)^{-\frac{2 N+2-i}{2}} C^{i+1} \\
& +\sum_{i=2}^{N+1} i a_{i-1}(N)(1-4 t)^{-\frac{2 N+2-i}{2}} C^{i+1} .
\end{aligned}
$$

On the other hand, replacing $N$ by $N+1$ in (2.4), we get

$$
C^{(N+1)}=\sum_{i=1}^{N+1} a_{i}(N+1)(1-4 t)^{-\frac{2 N+2-i}{2}} C^{i+1} .
$$

From (2.5) and (2.6), we can derive the following recurrence relations:

$$
\begin{aligned}
& a_{1}(N+1)=2(2 N-1) a_{1}(N), \\
& a_{N+1}(N+1)=(N+1) a_{N}(N),
\end{aligned}
$$

and

$$
a_{i}(N+1)=i a_{i-1}(N)+2(2 N-i) a_{i}(N),(2 \leq i \leq N) .
$$

In addition, from (2.2) and (2.4), we observe that

$$
a_{1}(1)(1-4 t)^{-\frac{1}{2}} C^{2}=C^{(1)}=(1-4 t)^{-\frac{1}{2}} C^{2} .
$$

Thus, by (2.10), we get

$$
a_{1}(1)=1
$$


In Below, for any positive integer $N,(2 N-1)$ !! will denote

$$
(2 N-1) ! !=(2 N-1)(2 N-3) \cdots 1 .
$$

From (2.7) and (2.11), we note that

$$
\begin{aligned}
a_{1}(N+1) & =2(2 N-1) a_{1}(N)=2^{2}(2 N-1)(2 N-3) a_{1}(N-1) \\
& =\cdots \\
& =2^{N}(2 N-1)(2 N-3) \cdots 1 a_{1}(1) \\
& =2^{N}(2 N-1) ! !
\end{aligned}
$$

and

$$
\begin{aligned}
a_{N+1}(N+1) & =(N+1) a_{N}(N)=(N+1) N a_{N-1}(N-1) \\
& =\cdots \\
& =(N+1) N \cdots 2 a_{1}(1)=(N+1) !
\end{aligned}
$$

In the following, we will use the notations:

$$
(x ; \alpha)_{n}=x(x-\alpha) \cdots(x-(n-1) \alpha), \quad \text { for } n \geq 1,
$$

and

$$
(x ; \alpha)_{0}=1 .
$$

For $i=2$ in (2.9), we have

$$
\begin{aligned}
a_{2}(N+1)= & 2 a_{1}(N)+2(2 N-2) a_{2}(N) \\
= & 2 a_{1}(N)+2(2 N-2)\left(2 a_{1}(N-1)+2(2 N-4) a_{2}(N-1)\right) \\
= & 2\left(a_{1}(N)+2(2 N-2) a_{1}(N-1)\right)+2^{2}(2 N-2)(2 N-4) a_{2}(N-1) \\
= & 2\left(a_{1}(N)+2(2 N-2) a_{1}(N-1)\right) \\
& +2^{2}(2 N-2)(2 N-4)\left(2 a_{1}(N-2)+2(2 N-6) a_{2}(N-2)\right) \\
= & \left.2\left(a_{1}(N)+2(2 N-2) a_{1}(N-1)\right)+2^{2}(2 N-2)(2 N-4) a_{1}(N-2)\right) \\
& +2^{3}(2 N-2)(2 N-4)(2 N-6) a_{2}(N-2) \\
= & \cdots \\
= & 2 \sum_{k=0}^{N-2} 2^{k}(2 N-2 ; 2)_{k} a_{1}(N-k)+2^{N-1}(2 N-2 ; 2)_{N-1} a_{2}(2) \\
= & 2 \sum_{k=0}^{N-1} 2^{k}(2 N-2 ; 2)_{k} a_{1}(N-k) .
\end{aligned}
$$

Proceeding analogously to the case of $i=2$, for $i=3$ and 4 , we obtain

$$
\begin{aligned}
& a_{3}(N+1)=3 \sum_{k=0}^{N-2} 2^{k}(2 N-3 ; 2)_{k} a_{2}(N-k), \\
& a_{4}(N+1)=4 \sum_{k=0}^{N-3} 2^{k}(2 N-4 ; 2)_{k} a_{3}(N-k) .
\end{aligned}
$$


A NEW APPROACH TO CATALAN NUMBERS USING DIFFERENTIAL EQUATIONS 5

Continuing this process, we can deduce that

$$
a_{i}(N+1)=i \sum_{k=0}^{N-i+1} 2^{k}(2 N-i ; 2)_{k} a_{i-1}(N-k), \quad \text { for } 2 \leq i \leq N .
$$

Now, we give explicit expressions for $a_{i}(N+1)(2 \leq i \leq N)$. From (2.13) and (2.16), we have

$$
\begin{aligned}
a_{2}(N+1) & =2 \sum_{k_{1}=0}^{N-1} 2^{k_{1}}(2 N-2 ; 2)_{k_{1}} a_{1}\left(N-k_{1}\right) \\
& =2 \sum_{k_{1}=0}^{N-1} 2^{k_{1}}(2 N-2 ; 2)_{k_{1}} 2^{N-k_{1}-1}\left(2 N-2 k_{1}-3\right) ! ! \\
& =2 ! 2^{N-1} \sum_{k_{1}=0}^{N-1}(2 N-2 ; 2)_{k_{1}}\left(2 N-2 k_{1}-3\right) ! !
\end{aligned}
$$

Also, from (2.17) and (2.20), we get

$$
\begin{aligned}
a_{3}(N+1)= & 3 \sum_{k_{2}=0}^{N-2} 2^{k_{2}}(2 N-3 ; 2)_{k_{2}} a_{2}\left(N-k_{2}\right) \\
= & 3 \sum_{k_{2}=0}^{N-2} 2^{k_{2}}(2 N-3 ; 2)_{k_{2}} 2^{N-k_{2}-1} \\
& \times \sum_{k_{1}=0}^{N-2-k_{2}}\left(2 N-2 k_{2}-4 ; 2\right)_{k_{1}}\left(2 N-2 k_{1}-2 k_{1}-5\right) ! ! \\
= & 3 ! 2^{N-2} \sum_{k_{2}=0}^{N-2} \sum_{k_{1}=0}^{N-2-k_{2}}(2 N-3 ; 2)_{k_{2}}\left(2 N-2 k_{2}-4 ; 2\right)_{k_{1}} \\
& \times\left(2 N-2 k_{1}-2 k_{1}-5\right) ! !
\end{aligned}
$$

Continuing this process, we can deduce that

$$
\begin{aligned}
a_{i}(N+1)=2^{N-i+1} i ! \sum_{k_{i-1}=0}^{N-i+1} \sum_{k_{i-2}=0}^{N-i+1-k_{i-1}} \cdots \sum_{k_{1}=0}^{N-i+1-k_{i-1}-\cdots-k_{2}}(2 N-i ; 2)_{k_{i-1}} \\
\\
\left(2 N-2 k_{i-1}-i-1 ; 2\right)_{k_{i-2}} \times \cdots \times\left(2 N-2 k_{i-1}-\cdots-2 k_{2}-2 i+2 ; 2\right)_{k_{1}} \\
\times\left(2 N-2 k_{i-1}-\cdots-2 k_{1}-2 i+1\right) ! ! \\
=2^{N-i+1} i ! \sum_{k_{i-1}=0}^{N-i+1} \sum_{k_{i-2}=0}^{N-i+1-k_{i-1}} \cdots \sum_{k_{1}=0}^{N-i+1-k_{i-1}-\cdots-k_{2}} \\
\quad \prod_{l=1}^{i-1}\left(2 N-2 \sum_{j=l+1}^{i-1} k_{j}-2 i+1+l ; 2\right)_{k_{l}} \times\left(2 N-2 \sum_{j=1}^{i-1} k_{j}-2 i+1\right) ! !,
\end{aligned}
$$

for $2 \leq i \leq N$.

Remark. We note here that (2.22) is also valid for $i=N+1$.

Therefore, from (2.13) and (2.22), we obtain the following theorem. 
Theorem 1. The family of differential equations

$$
C^{(N)}=\sum_{i=1}^{N} a_{i}(N)(1-4 t)^{-\frac{2 N-i}{2}} C^{i+1} \quad(N=1,2,3, \cdots)
$$

have a solution

$$
C=C(t)=\frac{2}{1+\sqrt{1-4 t}}
$$

where

$$
\begin{aligned}
a_{1}(N) & =2^{N-1}(2 N-3) ! ! \\
a_{i}(N) & =2^{N-i} i ! \sum_{k_{i-1}=0}^{N-i} \sum_{k_{i-2}=0}^{N-i-k_{i-1}} \cdots \sum_{k_{1}=0}^{N-i-k_{i-1}-\cdots-k_{2}} \\
& \times \prod_{l=1}^{i-1}\left(2 N-2 \sum_{j=l+1}^{i-1} k_{j}-2 i-1+l ; 2\right)_{k_{l}} \\
& \times\left(2 N-2 \sum_{j=1}^{i-1} k_{j}-2 i-1\right) ! !
\end{aligned}
$$

We recall that the Catalan numbers $C_{n}$ are defined by the generating funcion

$$
C=C(t)=\frac{2}{1+\sqrt{1-4 t}}=\sum_{n=0}^{\infty} C_{n} t^{n}
$$

More generally, the higher-order Catalan numbers $C_{n}^{(r)}$ of order $r$ are given by

$$
\left(\frac{2}{1+\sqrt{1-4 t}}\right)^{r}=\sum_{n=0}^{\infty} C_{n}^{(r)} t^{n}
$$

On the one hand, from (2.24), we have

$$
\begin{aligned}
C^{(N)} & =\sum_{n=N}^{\infty} C_{n}(n)_{N} t^{n-N} \\
& =\sum_{n=0}^{\infty} C_{n+N}(n+N)_{N} t^{n}
\end{aligned}
$$

where

$$
(x)_{n}=x(x-1) \cdots(x-n+1), \quad \text { for } n \geq 1,(x)_{0}=1 .
$$


On the other hand, by Theorem 1, we have

$$
\begin{aligned}
C^{(N)} & =\sum_{i=1}^{N} a_{i}(N)(1-4 t)^{-\frac{2 N-i}{2}} C^{i+1} \\
& =\sum_{i=1}^{N} a_{i}(N) \sum_{m=0}^{\infty}\left(\begin{array}{c}
\frac{2 N-i}{2}+m-1 \\
m
\end{array}\right) 4^{m} t^{m} \sum_{l=0}^{\infty} C_{l}^{(i+1)} t^{l} \\
& =\sum_{i=1}^{N} a_{i}(N) \sum_{n=0}^{\infty} \sum_{m=0}^{n} 4^{m}\left(\begin{array}{c}
\frac{2 N-i}{2}+m-1 \\
m
\end{array}\right) C_{n-m}^{(i+1)} t^{n} \\
& =\sum_{n=0}^{\infty}\left(\sum_{i=1}^{N} \sum_{m=0}^{n} 4^{m}\left(\begin{array}{c}
\frac{2 N-i}{2}+m-1 \\
m
\end{array}\right) a_{i}(N) C_{n-m}^{(i+1)}\right) t^{n}
\end{aligned}
$$

Comparing (2.26) with (2.28), we get the following Theorem.

Theorem 2. For $n=0,1,2, \cdots$, and $N=1,2,3, \cdots$,

$$
C_{n+N}=\frac{1}{(n+N)_{N}} \sum_{i=1}^{N} \sum_{m=0}^{n} 4^{m}\left(\begin{array}{c}
\frac{2 N-i}{2}+m-1 \\
m
\end{array}\right) a_{i}(N) C_{n-m}^{(i+1)}
$$

where $a_{i}(N)$ 's are as in Theorem 1 .

\section{Inverse differential equations associated with Catalan numbers}

Here we will derive "inverse" differential equations to the ones obtained in Section 2. With $C=C(t)$ as in (2.1), we have

$$
C^{(1)}=(1-4 t)^{-\frac{1}{2}} C^{2}
$$

and

$$
C^{2}=(1-4 t)^{\frac{1}{2}} C^{(1)} .
$$

Differentiating both sides of (3.2), we get

$$
2 C C^{(1)}=-2(1-4 t)^{-\frac{1}{2}} C^{(1)}+(1-4 t)^{\frac{1}{2}} C^{(2)} .
$$

Substituting (3.1), into (3.3), we obtain

$$
2 C^{3}=-2 C^{(1)}+(1-4 t) C^{(2)} .
$$

Differentiating both sides of (3.4), we have

$$
3 ! C^{2} C^{(1)}=-6 C^{(2)}+(1-4 t) C^{(3)}
$$

Substituting (3.1) into (3.5), we get

$$
3 ! C^{4}=-6(1-4 t)^{\frac{1}{2}} C^{(2)}+(1-4 t)^{\frac{3}{2}} C^{(3)} .
$$

So we are led to put

$$
N ! C^{N+1}=\sum_{i=0}^{\left[\frac{N}{2}\right]} b_{i}(N)(1-4 t)^{\frac{N}{2}-i} C^{(N-i)} \quad(N=1,2,3, \cdots) .
$$


Here $[x]$ denote the greatest integer not exceeding $x$. Differentiation of both sides of (3.7), gives

$$
\begin{aligned}
(N+1) ! C^{N} C^{(1)}= & \sum_{i=0}^{\left[\frac{N}{2}\right]}-4\left(\frac{N}{2}-i\right) b_{i}(N)(1-4 t)^{\frac{N}{2}-i-1} C^{(N-i)} \\
& +\sum_{i=0}^{\left[\frac{N}{2}\right]} b_{i}(N)(1-4 t)^{\frac{N}{2}-i} C^{(N+1-i)} \\
= & \sum_{i=1}^{\left[\frac{N}{2}\right]+1}-4\left(\frac{N}{2}+1-i\right) b_{i-1}(N)(1-4 t)^{\frac{N}{2}-i} C^{(N+1-i)} \\
& +\sum_{i=0}^{\left[\frac{N}{2}\right]} b_{i}(N)(1-4 t)^{\frac{N}{2}-i} C^{(N+1-i)} .
\end{aligned}
$$

Substituting (3.1) into (3.8), we obtain

$$
\begin{aligned}
(N+1) ! C^{N+1}= & \sum_{i=1}^{\left[\frac{N}{2}\right]+1}-4\left(\frac{N}{2}+1-i\right) b_{i-1}(N)(1-4 t)^{\frac{N+1}{2}-i} C^{(N+1-i)} \\
& +\sum_{i=0}^{\left[\frac{N}{2}\right]} b_{i}(N)(1-4 t)^{\frac{N+1}{2}-i} C^{(n+1-i)} .
\end{aligned}
$$

Also, by replacing $N$ by $N+1$ in (3.7), we get

$$
(N+1) ! C^{N+2}=\sum_{i=0}^{\left[\frac{N+1}{2}\right]} b_{i}(N+1)(1-4 t)^{\frac{N+1}{2}-i} C^{(N+1-i)} .
$$

Comparing (3.9) with (3.10), we have the following recurrence relations. Here we need to consider the even and odd cases of $N$ separately. The details are left to the reader.

$$
\begin{aligned}
& b_{0}(N+1)=b_{0}(N), \\
& b_{i}(N+1)=-4\left(\frac{N}{2}+1-i\right) b_{i-1}(N)+b_{i}(N), \quad \text { for } 1 \leq i \leq\left[\frac{N+1}{2}\right] .
\end{aligned}
$$

From (3.2) and (3.7), we have

$$
C^{2}=b_{0}(1)(1-4 t)^{\frac{1}{2}} C^{(1)}=(1-4 t)^{\frac{1}{2}} C^{(1)} .
$$

Thus, from (3.13), we get

$$
b_{0}(1)=1
$$

From (3.11), we easily obtain

$$
b_{0}(N+1)=b_{0}(N)=\cdots=b_{0}(1)=1 .
$$

The equation in (3.12) can be rewritten as

$$
b_{i}(N+1)=-2(N+2-2 i) b_{i-1}(N)+b_{i}(N)
$$

To proceed further, we define

$$
S_{N, 1}=N+(N-1)+\cdots+1,
$$


A NEW APPROACH TO CATALAN NUMBERS USING DIFFERENTIAL EQUATIONS 9

$$
S_{N, j}=N S_{N+1, j-1}+(N-1) S_{N, j-1}+\cdots+1 S_{2, j-1} \quad(j \geq 2) .
$$

Now,

$$
\begin{aligned}
b_{1}(N+1) & =-2 N b_{0}(N)+b_{1}(N) \\
& =-2 N+b_{1}(N) \\
& =-2 N-2(N-1)+b_{1}(N-1) \\
& =\cdots \\
& =-2(N+(N-1) \cdots+1)+b_{1}(1) \\
& =-2 S_{N, 1},
\end{aligned}
$$

and

$$
\begin{aligned}
b_{2}(N+1) & =-2(N-2) b_{1}(N)+b_{2}(N) \\
& =(-2)^{2}(N-2) S_{N-1,1}+b_{2}(N) \\
& =(-2)^{2}\left((N-2) S_{N-1,1}+(N-3) S_{N-2,1}\right)+b_{2}(N-1) \\
& =\cdots \\
& =(-2)^{2}\left((N-2) S_{N-1,1}+(N-3) S_{N-2,1}+\cdots+1 S_{2,1}\right)+b_{2}(3) \\
& =(-2)^{2} S_{N-2,2} .
\end{aligned}
$$

Similarly to the cases of $i=1$ and 2 , for $i=3$, we get

$$
b_{3}(N+1)=(-2)^{3} S_{N-4,3} .
$$

Thus we can deduce that, for $1 \leq i \leq\left[\frac{N+1}{2}\right]$,

$$
b_{i}(N+1)=(-2)^{i} S_{N+2-2 i, i} .
$$

Here, from (3.15) and (3.22), we obtain the following Theorem.

Theorem 3. The following family of differential equations

$$
N ! C^{N+1}=\sum_{i=0}^{\left[\frac{N}{2}\right]} b_{i}(N)(1-4 t)^{\frac{N}{2}-i} C^{(N-i)} \quad(N=1,2,3 \cdots)
$$

have a solution

$$
C=C(t)=\frac{2}{1+\sqrt{1-4 t}}
$$

where

$$
b_{0}(N)=1, b_{i}(N)=(-2)^{i} S_{N+1-2 i, i}, \quad\left(1 \leq i \leq\left[\frac{N}{2}\right]\right) .
$$


Now, we would like to give an application of the result in Theorem 3 From (3.23), we have the following

$$
\begin{aligned}
N ! \sum_{k=0}^{\infty} C_{k}^{(N+1)} t^{k}= & \sum_{i=0}^{\left[\frac{N}{2}\right]} b_{i}(N) \sum_{l=0}^{\infty}\left(\begin{array}{c}
\frac{N}{2}-i \\
l
\end{array}\right)(-4 t)^{l} \\
& \times \sum_{m=0}^{\infty} C_{m+N-i}(m+N-i)_{N-i} t^{m} \\
= & \sum_{i=0}^{\left[\frac{N}{2}\right]} b_{i}(N) \sum_{k=0}^{\infty} \sum_{m=0}^{k}\left(\begin{array}{c}
\frac{N}{2}-i \\
k-m
\end{array}\right)(-4)^{k-m} \\
& \times C_{m+N-i}(m+N-i)_{N-i} t^{k} \\
= & \sum_{k=0}^{\infty}\left(\sum_{i=0}^{\left[\frac{N}{2}\right]} \sum_{m=0}^{k}\left(\begin{array}{c}
\frac{N}{2}-i \\
k-m
\end{array}\right)(m+N-i)_{N-i}\right. \\
& \left.\times(-4)^{k-m} b_{i}(N) C_{m+N-i}\right) t^{k}
\end{aligned}
$$

Thus, from (3.24), we get the following Theorem.

Theorem 4. For $k=0,1,2 \cdots$, and $N=1,2,3 \cdots$, we have

$$
\begin{aligned}
C_{k}^{(N+1)}= & \frac{1}{N !} \sum_{i=0}^{\left[\frac{N}{2}\right]} \sum_{m=0}^{k}\left(\begin{array}{c}
\frac{N}{2}-i \\
k-m
\end{array}\right)(m+N-i)_{N-i} \\
& \times(-4)^{k-m} b_{i}(N) C_{m+N-i},
\end{aligned}
$$

where $b_{i}(N)^{\prime} s$ are as in Theorem 3 .

Remark. Combining (2.23) with (3.23), we can show that

$$
\begin{aligned}
(1-4 t)^{\frac{N}{2}} C^{N+1} & =\sum_{i=0}^{\left[\frac{N}{2}\right]} \sum_{j=1}^{N-i} \frac{a_{j}(N-i)}{N !} b_{i}(N)(1-4 t)^{\frac{j}{2}} C^{j+1} \\
& =\sum_{j=1}^{N} \sum_{i=0}^{\min \left(N-j,\left[\frac{N}{2}\right]\right)} \frac{a_{j}(N-i)}{N !} b_{i}(N)(1-4 t)^{\frac{j}{2}} C^{j+1} .
\end{aligned}
$$

Equivalently, (3.25) can be expressed as

$$
\sum_{i=0}^{\min \left(N-j,\left[\frac{N}{2}\right]\right)} \frac{a_{j}(N-i)}{N !} b_{i}(N)=\delta_{j, N}, \quad(1 \leq j \leq N),
$$

when $\delta_{j, N}$ is the Kronecker delta.

\section{FurTher REMARKS}

We start our discussion here with the following expansion of $\sqrt{1+y}$ :

$$
\sqrt{1+y}=\sum_{n=0}^{\infty}\left(\begin{array}{c}
2 n \\
n
\end{array}\right) \frac{(-1)^{n-1}}{4^{n}(2 n-1)} y^{n} .
$$


Integrating both sides of (4.1) from 0 to 1 , we immediately obtain

$$
\sum_{n=0}^{\infty} C_{n} \frac{(-1)^{n-1}}{4^{n}(2 n-1)}=\frac{1}{3}(4 \sqrt{2}-2)
$$

Next, we integrate the generating function of the Catalan numbers from 0 to $\frac{1}{4}$.

$$
\int_{0}^{\frac{1}{4}} \frac{2}{1+\sqrt{1-4 t}} d t=\int_{0}^{\frac{1}{4}} \sum_{n=0}^{\infty} \frac{1}{n+1}\left(\begin{array}{c}
2 n \\
n
\end{array}\right) t^{n} d t
$$

The left hand side of (4.3) is, after making the change of variable $t=\frac{1}{4}\left(1-y^{2}\right)$, equal to

$$
\int_{0}^{1} \frac{y}{1+y} d y=[y-\log (1+y)]_{0}^{1}=1-\log 2 .
$$

Thus, from (4.3) and (4.4), we get the following identity.

$$
\sum_{n=0}^{\infty} \frac{1}{(n+1)^{2}}\left(\begin{array}{c}
2 n \\
n
\end{array}\right)\left(\frac{1}{4}\right)^{n+1}=1-\log 2 .
$$

Finally, again from the generating function of Catan numbers and (4.1), we have

$$
\begin{aligned}
2 & =\left(\sum_{l=0}^{\infty} C_{l} t^{l}\right)(1+\sqrt{1-4 t}) \\
& =\left(\sum_{l=0}^{\infty} C_{l} t^{l}\right)\left(1-\sum_{m=0}^{\infty}\left(\begin{array}{c}
2 m \\
m
\end{array}\right) \frac{1}{2 m-1} t^{m}\right) \\
& =\sum_{l=0}^{\infty} C_{l} t^{l}-\left(\sum_{l=0}^{\infty} C_{l} t^{l}\right)\left(\sum_{m=0}^{\infty}\left(\begin{array}{c}
2 m \\
m
\end{array}\right) \frac{1}{2 m-1} t^{m}\right) \\
& =\sum_{n=0}^{\infty} C_{n} t^{n}-\sum_{n=0}^{\infty}\left(\sum_{m=0}^{n}\left(\begin{array}{c}
2 m \\
m
\end{array}\right) \frac{1}{2 m-1} C_{n-m}\right) t^{n} \\
& =\sum_{n=0}^{\infty}\left(C_{n}-\sum_{m=0}^{n} C_{m} C_{n-m} \frac{m+1}{2 m-1}\right) t^{n} .
\end{aligned}
$$

Therefore, from (4.6) we obtain the recurrence relation:

$$
C_{n}-\sum_{m=0}^{n} C_{m} C_{n-m} \frac{m+1}{2 m-1}= \begin{cases}2 & \text { if } n=0, \\ 0 & \text { if } n>0 .\end{cases}
$$

Noting that $C_{n}=\frac{1}{n}\left(\begin{array}{c}2 n \\ n+1\end{array}\right)$, we see that (4.7) for $n>0$ is equivalent to the following identity.

$$
\left(\begin{array}{c}
2 n \\
n+1
\end{array}\right)=n \sum_{m=0}^{n} \frac{m+1}{m(n-m)(2 m-1)}\left(\begin{array}{c}
2 m \\
m+1
\end{array}\right)\left(\begin{array}{c}
2 n-2 m \\
n-m+1
\end{array}\right), \quad(n>0) .
$$

Further, separating terms corresponding to $m=0$ and $m=n$ from (4.7) for $n>0$ and after rearranging the terms, we get the following recurrence relations for the Catalan numbers.

$$
C_{0}=C_{1}=1, \quad C_{n}=\frac{2 n-1}{3(n-1)} \sum_{m=1}^{n-1} C_{m} C_{n-m} \frac{m+1}{2 m-1}, \quad(n \geq 2) .
$$


Compare (4.9) with the recurrence relation in (1.2).

\section{REFERENCES}

[1] C. Adiga, H. Ariamanesh, Some properties of Cayley graphs on symmetric groups $S_{n}$, Int. J. Algebra 6 (2012), no. 17-20, 807-813.

[2] H. W. Gould, Sums and convolved sums of Catalan numbers and their generating functions, Indian J. Math. 46 (2004), no. 2-3, 137-160.

[3] R. Hampel, On the problem of Catalan, (Polish) Prace Mat. 4 (1960), 11-19.

[4] S. Hyyro, On the Catalan problem, (Finnish) Arkhimedes 1963 (1963), no. 1, 53-54.

[5] Y. He, C. Wang, Recurrence formulae for Apostol-Bernoulli and Apostol-Euler polynomials Adv. Difference Equ. 2012, 2012:209, 16 pp.

[6] K. Inkeri On Catalan's problem, Acta Arith. 9 (1964), 285-290.

[7] D. Kang, J. Jeong, S.-J. Lee, S.-H. Rim, A note on the Bernoulli polynomials arising from a non-linear differential equation, Proc. Jangjeon Math. Soc. 16 (2013), no. 1, 37-43.

[8] T. Kim, D. S. Kim, A note on nonlinear Changhee differential equations, Russ. J. Math. Phys. 23 (2016), 88-92.

[9] T. Kim, D. S. Kim, J.-J. Seo, H.-I. Kwon, Differnetial equations associated with $\lambda$-Changhee polynomials, J. Nonlinear Sci. Appl. 9 (2016), 3098-3111.

[10] J. Morgado, Some remarks on an identity of Catalan concerning the Fibonacci numbers, Special issue in honor of António Monteiro. Portugal. Math. 39 (1980), no. 1-4, 341-348 (1985).

[11] A. Natucci, Ricerche sistematiche intorno al "teorema di Catalan", (Italian) Giorn. Mat. Battaglini (5) 2(82) (1954), 297-300.

[12] R. Rangarajan, P. Shashikala, A pair of classical orthogonal polynomials connected to Catalan numbers, Adv. Stud. Contemp. Math. (Kyungshang) 23 (2013), no. 2, 323-335.

[13] A. Rotkiewicz, Sur le probléme de Catalan, (French) Elem. Math. 15 (1960), 121-124.

[14] A. D. Sands, On generalised Catalan numbers, Discrete Math. 21 (1978), no 2. 219-221.

[15] Y. Simsek, Identities associated with generalized Stirling type numbers and Eulerian type polynomials. Math. Comput. Appl. 18 (2013), no. 3, 251-263.

Department of Mathematics, Kwangwoon University, Seoul 139-701, Republic of KoREA

E-mail address: tkkim@kw.ac.kr

Department of Mathematics, Sogang University, Seoul 121-742, Republic of Korea

E-mail address: dskim@sogang.ac.kr 